\title{
Determination of Chlorobenzenes and Nitrobenzoates in Wastewater by Dispersive Liquid-Liquid Microextraction Coupled to Capillary Gas Chromatography
}

\author{
Jianqi Sun ${ }^{1,2, a}$ \\ ${ }^{1}$ College of Chemistry and Envioronmental Engineering, Jiujiang University, Jiujiang 332005, China \\ ${ }^{2}$ Poyang Lake Eco-economy Research Center, Jiujiang University, Jiujiang 332005, China \\ asunjianqi01@sina.com
}

Keywords: Chlorobenzenes, Nitrobenzoates, Capillary Gas Chromatography, Dispersive liquid-liquid Microextraction, Wastewater.

Abstract. A fast, eco-friendly and reliable method combining dispersive liquid-liquid microextracion (DLLME) with capillary gas chromatography (GC) was developed for the simultaneous determination of $p$-dichlorobenzene ( $p$-DCB), $o$-dichlorobenzene ( $o$-DCB), nitrobenze (NB), 1,2,4-trichlorobenzene (TCB), and 3-nitrotoluene (NT) in wastewater. To achieve this goal, dispersive liquid-liquid microextraction (DLLME) was applied as a sample preparation technique. The DLLME parameters such as the types and volume of extraction solvent, the types and volume of the disperser solvent, $\mathrm{pH}$ value and salt addition were investigated and optimized. The method was linear in the ranges from $2.2 \times 10^{-2}$ to $200 \mu \mathrm{g} \cdot \mathrm{mL}^{-1}$ for aforementioned compounds with $R^{2} \geq$ 0.9987. The DLLME procedure allowed efficient recovery of these compounds ranging between $89 \%$ and $104 \%$ with a relative standard deviation $(\mathrm{RSD}) \leq 9.6(\mathrm{n}=6)$ for genuine wastewater samples spiked with 10,50 and $100 \mu \mathrm{g} \cdot \mathrm{mL}^{-1}$ of standard substances, respectively. These results showed the potential of this technique for chlorobenzenes and nitrobenzoates monitoring in wastewater samples. Furthermore, the methods are simple, reproducible, and inexpensive.

\section{Introduction}

Chlorobenzenes and nitrobenzoates are extensively used during experiments and productions, and also are typically dangerous, explosive, flammable, toxic, and environmentally hazardous [1]. Therefore, the detection of these compounds is hence important which ensures the safety of human health and properties and also avoids environmental disasters. Numerous methodologies have been demonstrated to determination of chlorobenzenes and nitrobenzoates, such as gas chromatography-mass spectrometry (GC-MS) [2-4], high performance liquid chromatography-mass spectrometry (HPLC-MS) [5], sensors [1, 6, 7] etc.

Dispersive liquid-liquid microextraction (DLLME) has emerged recently as a new and very efficient alternative technique to concentrate target analytes dissolved in organic extracts, allowing a high enrichment factor in a simple and quick manner [8]. DLLME has been applied for determination of a vast variety of organic and inorganic compounds in different matrices $[9,10]$. In this paper, DLLME-GC was established for the determination of chlorobenzenes and nitrobenzoates in wastewater, and the features of this method were discussed in detail in the following sections.

\section{Experimental}

Apparatus and reagents. Measurements were carried out with Aglient $6890 \mathrm{~N}$ gas chromatograph fitted with a flame ionization detector (FID) (Agilent Corporation, America). Separation was performed on a HP-5 capillary column $(30 \mathrm{~m} \times 0.32 \mathrm{~mm}$ I. D. $\times 0.25 \mu \mathrm{m}$ film thickness). DLLME procedure was conducted in a $10 \mathrm{~mL}$ glass centrifugal tube.

Analytical grade standards of the compounds used in this work were obtained from Sinopharm Chemical Reagent Co., Ltd (Shanghai, China), which included $p$-DCB, $o$-DCB, NB, TCB, and NT. Dichloromethane, trichloromethane, carbon tetrachloride, acetone, acetonitrile and isooctane (Concord, Tianjin, China), were all HPLC grade solvents. The deionized water was purified with an Aquapro series water purification system (Chongqing, China). 
Stock solutions $\left(1000 \mu \mathrm{g} \cdot \mathrm{mL}^{-1}\right)$ were prepared in deionized water and stored in the fridge at $4{ }^{\circ} \mathrm{C}$. Working standard solutions of each compound were prepared by appropriate dilution of the stock solution using deionized water. The genuine wastewater samples collected from a local factory were filtered by using a $0.45 \mu \mathrm{m}$ membrane, and stored in amber bottles at $4{ }^{\circ} \mathrm{C}$ until analysis.

Chromatographic condition. The oven temperature program was as follows: the initial temperature $60^{\circ} \mathrm{C}$ increased to $100{ }^{\circ} \mathrm{C}$ (hold for $1 \mathrm{~min}$ ) at a rate of $20^{\circ} \mathrm{C} \cdot \mathrm{min}^{-1}$, and then to $140^{\circ} \mathrm{C}$ at $20^{\circ} \mathrm{C} \cdot \mathrm{min}^{-1}$. The injector temperature was $280{ }^{\circ} \mathrm{C}$ and the detector temperatures were $300{ }^{\circ} \mathrm{C}$. Nitrogen (99.999\%) was used as carrier and make-up gas at flow rates of 8.0 and $25 \mathrm{~mL} \cdot \mathrm{min}^{-1}$, respectively. The flow of air for FID was $350 \mathrm{~mL} \cdot \mathrm{min}^{-1}$ and flow rate of hydrogen was $30 \mathrm{~mL} \cdot \mathrm{min}^{-1}$. Two microliter was manually injected using the splitless mode.

Implementation of experiment. A $5.00 \mathrm{~mL}$ aqueous sample with its $\mathrm{pH}$ value adjusted to 6-12 in the presence of $10 \% \mathrm{NaCl}$, was placed into a $10 \mathrm{~mL}$ glass centrifugal tube. The mixture of 1.0 $\mathrm{mL}$ dichlormethane (as a disperser solvent) and $0.3 \mathrm{~mL}$ isooctane (as an extraction solvent) was rapidly added into the above-mentioned aqueous sample. After gently shaking for 3 min, the tube was centrifuged for $4 \mathrm{~min}$ at $6,000 \mathrm{rpm}$, then $2.0 \mu \mathrm{L}$ of the sedimented organic phase was withdrawn using a $10.0 \mu \mathrm{L}$ microsyringe (Agilent, America) and injected into the GC.

The linearity and linear range of the method were established using calibration curves obtained via the sextuplicate analysis of 5 compounds at six concentration levels $-10,20,40,80,120$ and $200 \mu \mathrm{g} \cdot \mathrm{mL}^{-1}$ in the standard working solutions. The limits of detection (LOD) were obtained from the analytical curves and calculated from the following expressions: LOD $=3 \sigma / \mathrm{s}$, where $\sigma$ is the standard deviation of the blank sample response $(n=20)$ and $s$ is the slope of the analytical curve.

The analysis of wastewater sample was complete in $8 \mathrm{~h}$. The concentrations of chlorobenzenes and nitrobenzoates in wastewater were determined. Then three different concentration levels were individually spiked to the wastewater samples and the recovery and precision of the method were obtained by assessing six replicates at each concentration.

\section{Results and discussion}

The optimization of DLLME conditions. There are several factors affecting the DLLME extraction process, including extraction solvent, disperser solvent, volume of the extraction solvent and disperser solvent, $\mathrm{pH}$ value of the aqueous solution, and the salting effect. The orthogonal test shows the optimized DLLME conditions: $0.3 \mathrm{~mL}$ isooctane (extractant), $1.0 \mathrm{~mL}$ dichlormethane (dispersant), $\mathrm{pH}=6-12$, and $10 \% \mathrm{NaCl}$ (salt).

Identification of the optimal chromatographic conditions. To separate these chlorobenzenes and nitrobenzoates as soon as possible and obtain normal peaks shapes, a lot of exploratory experiments were performed. As shown in Fig. 1a, under the optimized chromatographic conditions, $p$-DCB, $o$-DCB, NB, TCB, and NT were separated completely.
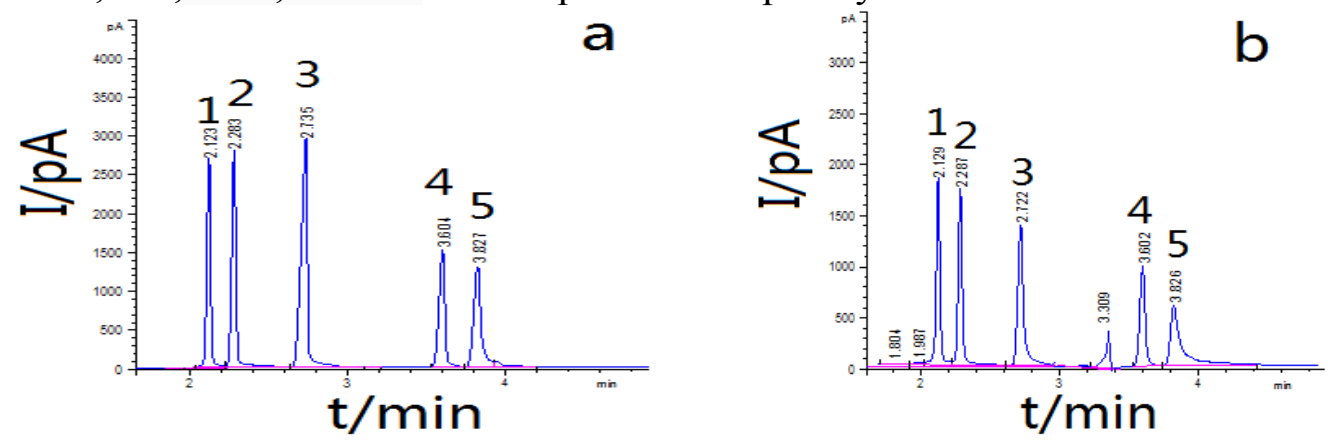

Fig. 1 Chromatograms of chlorobenzenes and nitrobenzoates in a standard solution (a) and an actual wastewater sample solution after extraction by DLLME (b) under the optimized GC-FID conditions. $p$-DCB (1), $o$-DCB (2), NB (3), TCB (4) and NT (5)

Linear range, calibration curve and limits of detection. Under the optimized GC-FID conditions, calibration curve, linear range and detection limits of chlorobenzenes and nitrobenzoates were listed in Table 1. Seen from Table 1, obviously, the detection limits of all five compounds were 
very low. The wide linear range (span four orders of magnitude) and good linearity $\left(R^{2} \geq 0.9987\right)$ showed high sensitivity and accuracy of this method.

Table 1 Linear range, Calibration curve and Detection limits

\begin{tabular}{cccc}
\hline Compounds & $\begin{array}{c}\text { Linear range } \\
\rho /\left(\mu \mathrm{g} \cdot \mathrm{mL}^{-1}\right)\end{array}$ & Calibration curve & $\begin{array}{c}\text { Detection limits } \\
\rho /\left(\mu \mathrm{g} \cdot \mathrm{mL}^{-1}\right)\end{array}$ \\
\hline$p$-DCB & $6.7 \times 10^{-2} \sim 200$ & $\mathrm{y}=18.242 \mathrm{x}+2.5334\left(\mathrm{R}^{2}=0.9988\right)$ & $2.0 \times 10^{-2}$ \\
$o$-DCB & $7.0 \times 10^{-2} \sim 200$ & $\mathrm{y}=18.515 \mathrm{x}-47.729\left(\mathrm{R}^{2}=0.9988\right)$ & $2.1 \times 10^{-2}$ \\
$\mathrm{NB}$ & $6.7 \times 10^{-2} \sim 200$ & $\mathrm{y}=22.258 \mathrm{x}-301.26\left(\mathrm{R}^{2}=0.9987\right)$ & $2.0 \times 10^{-2}$ \\
TCB & $7.3 \times 10^{-2} \sim 200$ & $\mathrm{y}=14.513 \mathrm{x}-156.60\left(\mathrm{R}^{2}=0.9991\right)$ & $2.2 \times 10^{-2}$ \\
$\mathrm{NT}$ & $8.0 \times 10^{-2} \sim 200$ & $\mathrm{y}=17.019 \mathrm{x}+551.25\left(\mathrm{R}^{2}=0.9987\right)$ & $2.4 \times 10^{-2}$ \\
\hline
\end{tabular}

Table 2 The average recoveries (percentage) of chlorobenzenes and nitrobenzoates and precision (\% RSD) of this method on spiked wastewater samples using DLLME procedures and GC-FID analysis $(\mathrm{n}=6)$

\begin{tabular}{ccccc}
\hline Compounds & $\begin{array}{c}\text { Added concetration } \\
\left(\mu \mathrm{g} \cdot \mathrm{mL}^{-1}\right)\end{array}$ & $\begin{array}{c}\text { Recovered concentration } \\
\left(\mu \mathrm{g} \cdot \mathrm{mL}^{-1}\right)\end{array}$ & $\begin{array}{c}\text { Recovery } \\
(\%)\end{array}$ & $\begin{array}{c}\text { RSD } \\
(\%)\end{array}$ \\
\hline \multirow{3}{*}{-DCB } & 10.0 & 9.63 & 96.3 & 9.1 \\
& 50.0 & 47.2 & 94.3 & 8.0 \\
$o$-DCB & 100.0 & 98.4 & 98.4 & 9.4 \\
& 10.0 & 9.62 & 96.2 & 9.1 \\
NB & 50.0 & 47.8 & 95.7 & 7.0 \\
& 100.0 & 98.3 & 98.3 & 5.8 \\
TCB & 10.0 & 9.76 & 97.6 & 9.6 \\
& 50.0 & 99.9 & 99.9 & 7.7 \\
NT & 100.0 & 98.6 & 99.6 & 7.0 \\
& 10.0 & 9.62 & 96.2 & 8.6 \\
& 50.0 & 98.8 & 98.8 & 7.2 \\
& 100.0 & 8.95 & 98.8 & 6.4 \\
& 10.0 & 50.2 & 89.5 & 9.7 \\
\hline
\end{tabular}

Determination of an actual wastewater sample. An genuine wastewater sample was manipulated at room temperature as described in the 'implementation of experiment' section. Fig. $1 \mathrm{~b}$ shows the separation of chlorobenzenes and nitrobenzoates in an actual sample after extraction by DLLME using the optimized GC-FID conditions. As shown, the separation of chlorobenzenes and nitrobenzoates in the genuine sample agreed with that of standard working solution well, indicating the validity of this method. Determined by applying external standard method, the concentration of $p$-DCB, $o$-DCB, NB, TCB, and NT in the actual sample was 10.69, 11.42, 12.70, 6.23 and 8.86 $\mu \mathrm{g} \cdot \mathrm{mL}^{-1}$, respectively.

Recovery and precision for the method. The recovery was determined by comparing the analytical response of the analytes in spiked samples before and after the extraction step, for three concentration levels $\left(10,50\right.$ and $\left.100 \mu \mathrm{g} \cdot \mathrm{mL}^{-1}\right)$, being each level performed six times. The mean recovery values ranged from 96.3 to $98.4 \%$ for $p$ - $\mathrm{DCB}$, from 95.7 to $98.3 \%$ for $o$-DCB, from 97.6 to $99.9 \%$ for NB, from 96.2 to $98.8 \%$ for TCB and from 89.5 to $103.5 \%$ for NT, as can be seen in Table 2. The precision expressed as relative standard deviation (\% RSD) was calculated from six replicates of spiked genuine wastewater samples at three concentration levels. The precision obtained ranged from 8.0 to $9.4 \%$ for $p$-DCB, from 5.8 to $9.1 \%$ for $o$-DCB, from 7.0 to $9.6 \%$ for NB, from 6.4 to $8.6 \%$ for TCB and from 6.9 to $9.7 \%$ for NT, as shown in Table 2 . 


\section{Conclusions}

In this study, DLLME combined with GC-FID has been successfully applied to the determination of chlorobenzenes and nitrobenzoates in wastewater samples. The DLLME technique demonstrated good analytical performance for the extraction of chlorobenzenes and nitrobenzoates from wastewater samples and proved to be time-saving, cheap and simple to perform.

Furthermore, the method presented good linearity, precision, recovery and accuracy. Therefore, it is clearly useful for monitoring chlorobenzenes and nitrobenzoates in wastewater samples.

\section{References}

[1] H. C. Liao, C.P. Hsu, M. C. Wu, C. F. Lu and W. F. Su: Anal. Chem. Vol. 85 (2013), p. 9305

[2] J. Y. Lee and H. K. Lee: Anal. Chem. Vol. 83 (2011), p. 6856

[3] S. H. Martín, I. Nijenhuis, H. H. Richnow and M. Gehre: Anal. Chem. Vol. 87 (2015), p. 951

[4] Y. Wang, Y. C. Kwok, Y. He and H. K. Lee: Anal. Chem. Vol. 70 (1998), p. 4610

[5] T. Gilevska, M. Gehre and H. H. Richnow: Anal. Chem. Vol. 86 (2014), p. 7252

[6] M. Faisal, S. B. Khan and M. M. Rahman: Appl. Surf. Sci. Vol. 258 (2012), p. 7515

[7] E. Katz, I. Willner and J. Wang: Electroanalysis, Vol. 16 (2004), p. 19

[8] S. C. Cunha, C. Cunha, A. R. Ferreira and J. O. Fernandes: Anal. Bioanal. Chem. Vol. 404 (2012), p.2453

[9] M. Rezaee, Y. Yamini, M. Faraji: J. Chromatogr. A Vol. 1217 (2010), p.2342

[10] E. Ranjbari and M. Hadjmohammadi: Talanta, Vol. 100 (2012), p. 447 